\title{
MATHEMATICAL MODELLING OF OSCILLATORY PROCESSES IN TRANSMISSION OF MOVEMENT OF AN ELECTRIC DRIVE WITH NON-LINEAR LONG ELASTIC ELEMENTS
}

\author{
Andriy Chaban, Tomasz Perzyński* \\ and Humanities in Radom, Radom, Poland \\ *E-mail of corresponding author: t.perzynski@uthrad.pl
}

Faculty of Transport, Electrical Engineering and Computer Science, Kazimierz Pulaski University of Technology

\section{Resume}

Mathematical model of transmission of movement of an electric drive system that includes long elastic elements, including the non-linear relation between tensors of strength and deformation is presented in this article. Mentioned type of transmission is applied in the tasks related to special-purpose transport. A method that is based on integral modification of variational HamiltonOstrogradsky principle was applied for the presented model. Results of the computer simulation of oscillatory processes in transmission of movement of an electromechanical system are presented in the article.

Available online: https://doi.org/10.26552/com.C.2021.2.C54-C64

\section{Article info}

Received 19 March 2020

Accepted 10 August 2020

Online 25 February 2021

\section{Keywords:}

modelling of transport systems, Hamilton-Ostrogradsky principle, mathematical modelling, systems of distributed parameters, computer simulation

ISSN 1335-4205 (print version) ISSN 2585-7878 (online version)

\section{Introduction}

Mathematical modelling of unspecified processes in the dynamical systems is one of the most topical tasks of an analysis of complicated technical objects. The electric drive systems that are used in special-purpose transport systems have become a part of such process of modelling. These systems may have long shafts, analysed as long elastic elements of distributed mechanical parameters [1-2]. Modelling and analysis of such elements makes it necessary to map a model in a prototypical system. It is particularly important in the event that concealed movements are included what extends a model and complicates output equations of the state of a system [3-4]. Using the modelling of complex system states allows to analyse it more precisely and to resign from developing a prototype of a system [5]. Regardless of the type of the analyzed system, computer simulation carried out based on the mathematical apparatus allows to determine the parameters affecting the operation of the system [6-7]. It also allows for analysis of different event scenarios [8-9]. An example of use of simulation to analyze a complex special-purpose transport system is presented in the work [10].

An analysis of transmission of the electric drives' movement, including long shafts of linear relation between tensors of strength and deformation was presented in the articles [5, 11-12]. In the real tasks of applied mechanics, the assumption, for example, in [4], is not always correct. Such analysis is applied to high-power drive systems that often work in hard, diverse conditions (special-purpose transport tasks - cranes, locomotives) [3, 13-14]. In such systems, the situations may emergel in which the linear relation between mentioned tensors is disrupted [15]. Then, the problem emerges how to include mentioned non-linear physical processes in an equation of the shaft. An attempt to solve this problem was made, for example, in the work [15]. Therefore, there is a need to include the non-linear mechanical processes using the non-linear elements in an equation of the shaft [15-16]. Another serious problem must be taken into account. If normal wave equation of torsional oscillations of a long shaft of linear distributed parameters has stable analytical solutions (e.g. Fourier method [17]), then in the case of the non-linear relations, analytical approach can be used only in some cases. To find a function of continuum of the shaft, numerical methods must be applied to solve boundary-value and mixed problems [5, 11-13].

Based on interdisciplinary modified HamiltonOstrogradsky method by extending Lagrangian function [5, 18-20], mathematical model of transmission of movement of a drive system is presented in the article. The goal of the article was mathematical modelling of unspecified processes in a system of transmission of movement of electric drives that includes non-linear elastic elements of 
a long shaft, analysed as a system of distributed mechanical parameters. To solve equations in the article, the elements of applied mathematics were also presented.

\section{Mathematical model of elastic long shaft of distributed parameters}

To develop a mathematical model of transmission of movement, modified variation method was applied, which is based on modification of the Hamilton-Ostrogradsky principle [5], by extending Lagrangian function with two elements [16, 21]. The first element includes energy of dissipation forces and the second one - energy of external forces of non-potential character. Similar approach was suggested in an article [18], in which two mentioned additional elements were added formally to a conservative Lagrangian, whereas, in another article [5], adding these elements was justified mathematically. In addition, a method presented in [5] allows to analyse dynamical systems of not only lumped parameters, but also to analyse unspecified processes in the complicated dynamical systems of both lumped [22] and distributed parameters [1,2].

Extended action functional according to HamiltonOstrogradsky is the following [5, 23]:

$S=\int_{t_{1}}^{t_{2}}\left(L^{*}+\int_{l} L_{l} d l\right) d t, I=\int_{l} L_{1} d l$

where:

$S$ - extended action functional according to HamiltonOstrogradsky,

$I$ - internal energy functional $[5,16,21]$,

$L^{*}$ - modified Lagrangian function [5, 19],

$L_{l}$ - linear density of modified Lagrangian function [5].

Extended Lagrangian function and its linear density are the following:

$$
\begin{aligned}
L^{*} & =T^{*}-P^{*}+\Phi^{*}-D^{*}, \\
L_{l} & =T_{l}-P_{l}+\Phi_{l}-D_{l},
\end{aligned}
$$

respectively, where:

$T^{*}\left(\tilde{T}^{*}\right)$ - kinetic energy (coenergy),

$P^{*}$ - conservative energy,

$\Phi^{*}$ - dissipation energy,

$D^{*}$ - energy of external forces of non-potential character.

Linear densities of mentioned functions are marked by subscript $l[5]$.

Since the model of a shaft is analysed as a system of only distributed parameters, then the modified action functional (S), based on Equation (1) can be written in the following form:

$$
S=\int_{t_{1}}^{t_{2}} \int_{l}\left(\tilde{T}_{l}-P_{l}+\Phi_{l}-D_{l}\right) d l d t,
$$

where:

$T_{v}, P_{l}, \Phi_{l}, D_{l}$ - appropriate linear densities of energy functions.

To obtain an equation of movement of the shaft, an equation of the extremals of a functional - Equation (3) [18,
21] should be calculated; therefore, variation of internal functional, see Equation (1), with subsequent comparison to zero is searched for. Then, it looks in the following way $[16,21]$ :

$\delta I=\delta \int_{l}\left(\tilde{T}_{l}-P_{l}+\Phi_{l}-D_{l}\right) d l=0$.

In order to find variation, the following procedures are applied: Gauss-Ostrogradsky theorem, rule of integration by parts, as well as assumption that the order of differentiation can be changed, because these procedures are independent [18-19]. Therefore, mechanical system may be analysed as a system of infinite degrees of freedom, which is a counterbalance to the systems of lumped parameters, in which the number of degrees of freedom is always determined [1, 2, 5, 15, 19].

An equation of extremals of this functional is the so-called Euler-Poisson equation [16]:

$$
\begin{aligned}
& \frac{\partial L}{\partial q}-\frac{\partial}{\partial t}\left(\frac{\partial L}{\partial q_{t}}\right)-\frac{\partial}{\partial x}\left(\frac{\partial L}{\partial q_{x}}\right)+\frac{\partial^{2}}{\partial x^{2}}\left(\frac{\partial L}{\partial q_{x x}}\right)+ \\
& +\frac{\partial^{2}}{\partial t^{2}}\left(\frac{\partial L}{\partial q_{t t}}\right)+\frac{\partial^{2}}{\partial x \partial t}\left(\frac{\partial L}{\partial q_{x t}}\right)=0,
\end{aligned}
$$

where:

$$
\begin{aligned}
\frac{\partial q}{\partial t} & \equiv q_{t}, \frac{\partial q}{\partial x} \equiv q_{x}, \frac{\partial^{2} q}{\partial x^{2}} \equiv q_{x x}, \\
\frac{\partial^{2} q}{\partial t^{2}} & \equiv q_{t t}, \frac{\partial^{2} q}{\partial x \partial t} \equiv q_{x t} .
\end{aligned}
$$

In such a case, for the systems of distributed parameters, adding specific coordinates is not possible. Therefore, the so-called function of generalized coordinates is added $q(x, t)$ and its generalized velocity $q_{t}(x, t)$. In the case of a long shaft $q(x, t) \equiv \varphi(x, t)$ - function of the shaft rotation angle and $q_{t}(x, t)=\omega(x, t)$ - function of rotational speed [5].

Therefore, system of elastic shaft can be presented using a function of two variables $(x, t), x$ - spatial coordinate along continuum of the shaft and $t$ - time coordinate.

Elements of the linear densities of extended Lagrangian function are presented [5, 15]:

$$
\begin{aligned}
& \frac{\partial T^{*}}{\partial x} \equiv T_{l}=\frac{\rho J_{P}}{2}\left(\frac{\partial \varphi}{\partial t}\right)^{2}, \\
& \frac{\partial P^{*}}{\partial x} \equiv P_{l}=\frac{G(\Delta \varphi) J_{P}}{2}\left(\frac{\partial \varphi}{\partial x}\right)^{2}, \\
& \frac{\partial \Phi^{*}}{\partial x} \equiv \Phi_{l}=\int_{0}^{t} \frac{\xi(\Delta \varphi)}{2}\left(\frac{\partial^{2} \varphi}{\partial x \partial t}\right)_{t=\tau}^{2} d \tau, \\
& \frac{\partial D^{*}}{\partial x} \equiv D_{l}=0,
\end{aligned}
$$

where:

$\rho$ - density of the shaft's material,

$J_{p}$ - polar moment of inertia of a connecting element, $G(\Delta \varphi)$ - shear modulus that depends on value of torsion between the shaft's elements,

$\xi(\Delta \varphi)$ - coefficients of internal dispersion in the shaft that also depend on value of torsion between elements of the shaft. 


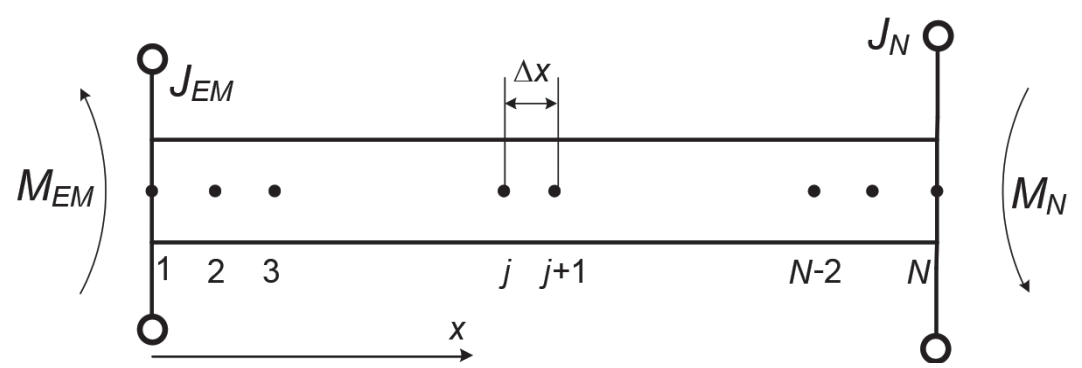

Figure 1 Diagram of a long shaft of transmission of movement of an electric drive

Assuming that $q \equiv \varphi, q_{t} \equiv \omega$, modified Lagrangian function will be the following:

$$
L=\frac{\rho J_{P}}{2} q_{t}^{2}-\frac{J_{P} G(\Delta \varphi)}{2} q_{x}^{2}+\frac{1}{2} \int_{0}^{t} \xi(\Delta \varphi) q_{x t \mid t=\tau}^{2} d \tau
$$

Since:

$\frac{\partial L}{\partial q} \equiv 0, \frac{\partial^{2}}{\partial x^{2}}\left(\frac{\partial L}{\partial q_{x x}}\right) \equiv 0, \frac{\partial^{2}}{\partial t^{2}}\left(\frac{\partial L}{\partial q_{t t}}\right) \equiv 0$,

Equation (5) is simplified to the following form:

$-\frac{\partial}{\partial t}\left(\frac{\partial L}{\partial q_{t}}\right)-\frac{\partial}{\partial x}\left(\frac{\partial L}{\partial q_{x}}\right)+\frac{\partial^{2}}{\partial x \partial t}\left(\frac{\partial L}{\partial q_{x t}}\right)=0$.

Giving an Equation (9) to Equation (11), changing the order of differentiation (derivatives from the functions of generalized coordinates are determined and uninterrupted), as well as applying derivative theorem from an integral beyond its upper boundary, one can write:

$-\frac{\partial}{\partial t} \frac{\partial L}{\partial q_{t}}=-\frac{\rho J_{P}}{2} \frac{\partial}{\partial t} \frac{\partial}{\partial q_{t}} q_{t}^{2}=-\rho J_{P} q_{t t}^{2}$,

$-\frac{\partial}{\partial x} \frac{\partial L}{\partial q_{x}}=-\frac{J_{P}}{2} \frac{\partial}{\partial x} \frac{\partial}{\partial q_{x}}\left[-G(\Delta \varphi) q_{x}^{2}\right]=$

$=J_{P} \frac{\partial}{\partial x}\left[G(\Delta \varphi) q_{x}\right]$,

$$
\begin{aligned}
& \frac{\partial^{2}}{\partial x \partial t} \frac{\partial L}{\partial q_{x t}}=\frac{1}{2} \frac{\partial}{\partial x} \frac{\partial^{2}}{q_{x t}} \frac{\partial}{\partial t}\left[\int_{0}^{t}\left(\xi(\Delta \varphi) q_{x t}\right)_{t=\tau}^{2} d \tau\right]= \\
& =\frac{1}{2} \frac{\partial}{\partial x} \frac{\partial^{2}}{q_{x t}}\left[\xi(\Delta \varphi) q_{x t}\right]^{2}=\frac{\partial}{\partial x}\left[\xi(\Delta \varphi) q_{x t}\right] .
\end{aligned}
$$

Summing up Equations (12 - 14), including relations: $q \equiv \varphi, q_{t} \equiv \omega$, finally equation of the shaft of non-linear elastic and dissipative elements are obtained:

$$
\begin{aligned}
& -\rho J_{P} \frac{\partial^{2} \varphi}{\partial t^{2}}+J_{P} \frac{\partial}{\partial x}\left[G(\Delta \varphi)\left(\frac{\partial \varphi}{\partial x}\right)\right]+ \\
& +\frac{\partial}{\partial x}\left[\xi(\Delta \varphi)\left(\frac{\partial^{2} \varphi}{\partial x \partial t}\right)\right]=0
\end{aligned}
$$

In a theory of non-linear applied mechanics, following assumptions are widely applied [15, 17, 24]:

$$
\begin{aligned}
G(\Delta \varphi)\left(\frac{\partial \varphi}{\partial x}\right) & \rightarrow G\left(\frac{\partial \varphi}{\partial x}\right)^{v+1}, \\
\xi(\Delta \varphi)\left(\frac{\partial^{2} \varphi}{\partial x \partial t}\right) & \rightarrow \xi\left(\frac{\partial^{2} \varphi}{\partial x \partial t}\right)^{\mu+1},
\end{aligned}
$$

where coefficients are written in the following way [15]:

$$
\begin{aligned}
& v=\frac{2 n_{1}-1}{2 m_{1}-1}, \mu=\frac{2 n_{2}-1}{2 m_{2}-1}, \\
& n_{1}, n_{2}, m_{1}, m_{2} \in N,
\end{aligned}
$$

here: $N$-natural number.

Mentioned coefficients are calculated based on experimental data, including Equation (17), [15]. Assuming additionally that coefficients $G(\Delta \varphi)$ and $\xi(\Delta \varphi)$ do not depend on a coordinate $x$, an equation of a long shaft can be written in the following way:

$$
\frac{\partial^{2} \varphi}{\partial t^{2}} \frac{\partial}{\partial x}\left[\frac{G}{\rho}\left(\frac{\partial \varphi}{\partial x}\right)^{v+1}\right]+\frac{\partial}{\partial x}\left[\frac{\xi}{\rho J_{P}}\left(\frac{\partial^{2} \varphi}{\partial x \partial t}\right)^{\mu+1}\right],
$$

or presented in the form:

$$
\begin{aligned}
& \frac{\partial^{2} \varphi}{\partial t^{2}}=\frac{G}{\rho}(v+1)\left(\frac{\partial \varphi}{\partial x}\right)^{v} \frac{\partial^{2} \varphi}{\partial t^{2}}+ \\
& +\frac{\xi}{\rho J_{P}}(\mu+1)\left(\frac{\partial^{2} \varphi}{\partial x \partial t}\right)^{\mu} \frac{\partial^{3} \varphi}{\partial x^{2} \partial t} .
\end{aligned}
$$

Therefore, consider the general case of a long shaft, as an element of transmission of movement of electric drives. The shaft is connected with a driving motor and load mechanism, Figure 1.

The following marks were used in Figure 1: $M_{E M}$ - electromagnetic moment of a driving motor, $M_{N}$ - load moment of a drive, $J_{E M}, J_{N}$ - moments of inertia of an engine rotor and load system, respectively, $x$ - spatial coordinate, $N$ - the number of the nodes of discretization of Equation (19), $\Delta x$ - step of discretization of spatial derivatives.

The boundary conditions for Equation (19) are based on equality of electromagnetic moments (or load) and elasticity and dissipation in the ends of the shaft in accordance with d'Alembert principle), [5, 17]

$$
\begin{aligned}
& \left.J_{E M} \frac{\partial^{2} \varphi}{\partial t^{2}}\right|_{x=0}-\left.G J_{P}\left(\frac{\partial \varphi}{\partial x}\right)^{v+1}\right|_{x=0}- \\
& -\left.\xi\left(\frac{\partial \varphi}{\partial x}\right)^{\mu+1}\right|_{x=0}=M_{E M},
\end{aligned}
$$

$$
\begin{aligned}
& \left.J_{N} \frac{\partial^{2} \varphi}{\partial t^{2}}\right|_{x=1}+\left.G J_{P}\left(\frac{\partial \varphi}{\partial x}\right)^{v+1}\right|_{x=L}+ \\
& +\left.\xi\left(\frac{\partial \varphi}{\partial x}\right)^{\mu+1}\right|_{x=L}=M_{N} .
\end{aligned}
$$


In order to simplify it, it was assumed that in the ends of the shaft, external moments are equal to specific moments of elasticity. Therefore, the following equations can be written:

$G J_{P} \frac{\Delta \varphi_{1}}{\Delta x}=M_{E M}, G J_{P} \frac{\Delta \varphi_{N}}{\Delta x}=-M_{N}$

where, see Figure 1:

$\Delta \varphi_{1}=\varphi_{2}-\varphi_{1}, \Delta \varphi_{N}=\varphi_{N}-\varphi_{N-1}$

Assuming that $\Delta x=L / 2$, boundary conditions can be written in the following way (angle of torsions of transmission):

$\left.\varphi(x, t)\right|_{x=0}=\frac{L M_{E M}}{2 G J_{P}}=a=$ const,

$\left.\varphi(x, t)\right|_{x=l}=\frac{L M_{N}}{2 G J_{P}}=-a=$ const.

Due to the fact that the shaft is inhibited, use of boundary conditions in Equations (24) and (25) to examine non-linear shaft is not sufficient. Therefore, two types of boundary conditions should be used. In the first case, the conditions are analysed using the Heaviside function [17]:

$. a_{1}(t)=a \cdot 1(t)$

From the point of view of applied physics, the use of such conditions means that the shaft was twisted and stabilized in such a state. Only mechanical wave moves towards the shaft, whereas, the ends of the shaft are immovable, which, with reference to applied mechanics, does not provide sufficient information about dynamics of the shaft.

In the second case, boundary conditions are analysed as a Dirac function:

$a_{2}(t)=\frac{d a_{1}(t)}{d t}=a \frac{d 1(t)}{d t}=a \delta(t)$.

Equation (27) should be understood as an impulse of finite value of amplitude $a$. From the point of view of applied physics, use of the boundary conditions in Equation (27) means that the shaft was twisted in $t=t-0$, and in a time moment $t=t+0$ - the ends of the shaft were quickly relieved. Therefore, not only that the mechanical wave is moving in the shaft, just like in the previous cause, but the elements of the shaft also rotate. It is interesting from the point of view of applied mechanics, providing information about dynamics of movement.

To solve Equation (19), simple method was applied [5].

For the conditions of the first type, see Equation (26), discrete system of normal non-linear differential equations is the following:

$\varphi_{1}=a, \omega_{1}=0, \varphi_{N}=-a, \omega_{N}=0$,

$$
\begin{aligned}
& \frac{d \omega_{j}}{d t}=(v+1) \frac{G}{\rho} \frac{\varphi_{j-1}-2 \varphi_{j}+\varphi_{j+1}}{(\Delta x)^{2}} \times \\
& \times\left[\frac{\varphi_{j+1}-\varphi_{j-1}}{2 \Delta x}\right]^{v}+(\mu+1) \frac{\xi}{\rho J_{p}} \times \\
& \times \frac{\omega_{j-1}-2 \omega_{j}+\omega_{j+1}}{(\Delta x)^{2}}\left[\frac{\omega_{j+1}-\omega_{j-1}}{2 \Delta x}\right]^{\mu},
\end{aligned}
$$

$\frac{d \varphi_{j}}{d t}=\omega_{j}, j=2,3 \ldots, N-1$.

For the boundary conditions of the second type, see Equation (27), system of equations is the following:

$$
\begin{aligned}
& \frac{d \omega_{1}}{d t}=\frac{M_{E M}-c\left(\varphi_{1}-\varphi_{2}\right)-v\left(\omega_{1}-\omega_{2}\right)}{J_{E M}+J_{1}}, \\
& \frac{d \omega_{j}}{d t}=(v+1) \frac{G}{\rho} \frac{\varphi_{j-1}-2 \varphi_{j}+\varphi_{j+1}}{(\Delta x)^{2}} \times \\
& \times\left[\frac{\varphi_{j+1}-\varphi_{j-1}}{2 \Delta x}\right]^{v}+(\mu+1) \frac{\xi}{\rho J_{p}} \times \\
& \times \frac{\omega_{j-1}-2 \omega_{j}+\omega_{j+1}}{(\Delta x)^{2}}\left[\frac{\omega_{j+1}-\omega_{j-1}}{2 \Delta x}\right]^{\mu}, \\
& j=2,3, \ldots N-1, \\
& \frac{d \omega_{N}}{d t}=\frac{-M_{N}-c\left(\varphi_{N}-\varphi_{N-1}\right)-v\left(\omega_{N}-\omega_{N-1}\right)}{J_{N}+J_{N-1}},
\end{aligned}
$$

$\frac{d \varphi_{j}}{d t}=\omega_{j}, j=1,2 \ldots, N-1, N$,

where:

$J_{1}=J_{N}=\rho J_{P} \Delta x, c=G J_{P} \Delta x, v=\xi J_{P} \Delta x$

Common integration is subject to the following nonlinear system of differential equations:

- for the first experiment: Equations (28) - (30),

- for the second experiment: Equations (31) - (35).

\section{The computer simulation results}

Computer simulation was conducted for a long transmission shaft with the following parameters: length of the shaft $L=6 \mathrm{~m}$, diameter $\mathrm{D}=0.15 \mathrm{~m}$, number of the nodes of discretization $N=90, G=8.1 \cdot 10^{10} \mathrm{~N} \cdot \mathrm{m}, \rho=7850 \mathrm{~kg} / \mathrm{m}^{3}$, $\xi=0.5 \mathrm{~N} \cdot \mathrm{m}^{2} \cdot \mathrm{s}, \Delta x=0.0667 \mathrm{~m}, J_{E M}=J_{N}=20 \mathrm{~N} \cdot \mathrm{m}^{2}$.

Two identical flywheels, of a moment of inertia $J=20$ $\mathrm{N} \cdot \mathrm{m}^{2}$, were mounted at the ends of the shaft. To activate dynamical system, the ends of the shaft twisted to initial values $\left.\varphi(x, t)\right|_{x=0}=0.2 \mathrm{rad},\left.\varphi(x, t)\right|_{x=L}=-0.2 \mathrm{rad}$, $v=0.2, \mu=0.1$, were calculated based on the comparative experiments [15].

Two experiments were conducted, in which the type of boundary conditions was changing. In the first experiment, mentioned conditions were considered as a Heaviside 


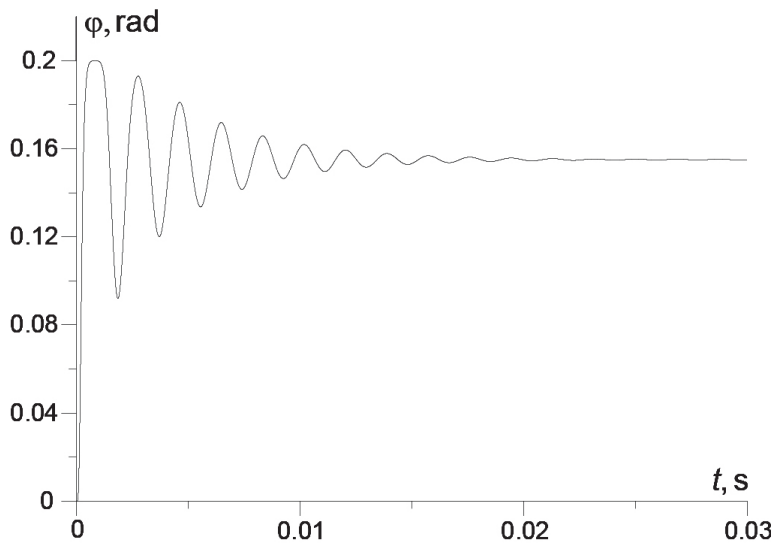

Figure 2 Angle of torsion of the shaft in the point of discretization $N o=9(0.6 \mathrm{~m}$ from the left end of the shaft $)$ in terms of time (linear variant)

function, whereas, in the second experiment, the conditions were considered as a Dirac function. From the point of view of applied electromechanics, description of the experiment is the following: in the first experiment, the ends of the shaft were twisted, fixed and left in such a state, in the second experiment, the ends of the shaft were released after torsion, which caused angular motion of the shaft. The shaft both in linear and non-linear cases was analysed in the research.

Figures 2 and 3 show angle of torsion of the shaft in the point of discretization $\mathrm{No}=9$ (see Figure 1), in linear (Figure 2) and non-linear (Figure 3) cases, in a function of time.

Therefore, the comparative analysis of descriptions from Figures 2 and 3 can be conducted:

- frequency of oscillation of the torsion angle function in the linear case is by about $20 \%$ higher in comparison to non-linear case: $f_{\text {lin }} \approx 500 \mathrm{~Hz}$, a $f_{\text {nonlin }} \approx 400 \mathrm{~Hz}$.

- time of vanishing of oscillation in the first case is about $0.02 \mathrm{~s}$, and in the second case - about $0.015 \mathrm{~s}$,

- maximal difference of amplitudes for the linear case is about $\Delta \varphi_{\max }^{\text {lin }} \approx 0.11 \mathrm{rad}$, and - $\Delta \varphi_{\max }^{\text {nonlin }} \approx 0.08 \mathrm{rad}$ for the non-linear case.

Since the shaft practically does not rotate, one must ask what physical rules are the background of the mentioned phenomena? The answer to this questions should be searched based only on the theory of mechanical field of analytical mechanics of a system continuum [15]. Mechanical wave moves along transmission of movement and dispersion forces makes it vanishing. In the linear case, one does not include non-linear relation between tensors of deformation and elongation and tensors of internal dispersion and elongation, which causes a bit incorrect physical form of the wave motion along the shaft. Conducting an experiment when the ends of the shaft are fixed, allows to conclude that the simulation results reflect the impact of the real non-linear properties of elastic and dissipative material on the work of the transmission elements of the complicated drives movement.

Figure 4 shows a transition function of the shaft's torsion velocity in the point of discretization No $=9(0.6 \mathrm{~m}$ from the left end of movement transmission). Figures 4 and

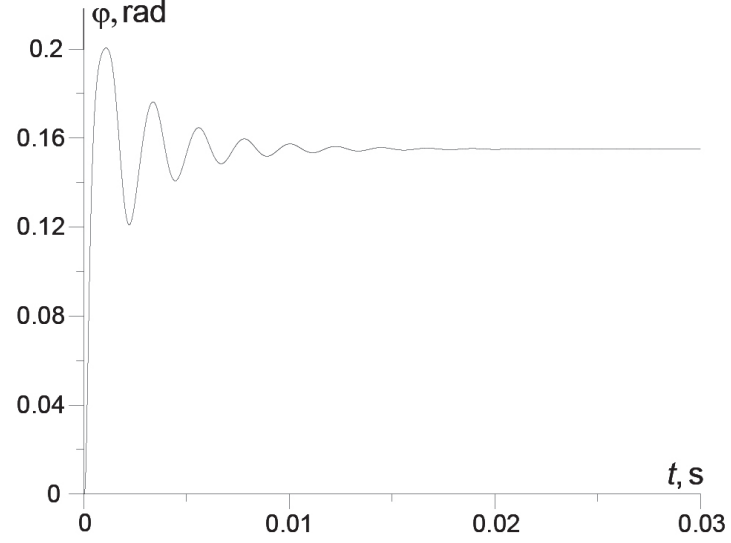

Figure 3 Angle of torsion of the shaft in the point of discretization $N o=9(0.6 \mathrm{~m}$ from the left end of the shaft $)$ in terms of time (non-linear variant)

3 are interrelated, because the time derivative as a function of angle presents a function of angular velocity. The impact of the non-linear wave processes in the shaft can also be observed in this case, which results in various frequencies between the linear and non-linear variants. Within about $15 \mathrm{~ms}$, the dissipation forces make mechanical wave practically vanishing. The shaft was twisted, which means that there is still potential energy in it.

Figure 5 shows the graphs of a function of moment of elasticity in the shaft in its beginning from the left side between the points of discretization No 3 - 4 - line 1 and in its central part: No 45 - 46 - line 2. From the point of view of applied physics, Figure 5 is of informative character because it demonstrates the wave processes in a continuum of the shaft. If in the beginning of the shaft, moment of elasticity reaches maximal value of amplitude of about $0.9 \mathrm{MN} \mathrm{m}$, then, in the central part, moment of elasticity in the shaft is equal to zero. The shock step moments of torsion cause formation of mechanical wave that moves from both ends of the shaft towards the centre. The velocity of this wave is limited. Therefore, in the center of the shaft, moment of elasticity decreased to zero within $0.5 \mathrm{~ms}$. It is obvious that oscillations of both functions are in a counterphase. Within $15 \mathrm{~ms}$, in steady state, moment of elasticity assumes constant value of about $0.3 \mathrm{MN} \mathrm{m}$. It should be added that time of stopping the mechanical wave is connected with time of counterphase of both functions.

Figures 6 - 9 show functional relations of an angle of torsions and velocity of torsion of the continuum elements of a shaft as a function of its length (spatial coordinate) for two time points: $0.2 \mathrm{~ms}$ and $2 \mathrm{~ms}$. An analysis of these functions should be considered comparing Figures 6 and 8 and 7 and 9 . Within $t=0.2 \mathrm{~ms}$, one can clearly see how mechanical wave starts moving along the shaft. The end parts of the shaft were twisted, whereas, the central part of the shaft remained immovable. Therefore, there was no phenomenon of change of discrete angles in a central part of the shaft (see Figure 1). This means that within $t=0.2$ $\mathrm{ms}$, elastic wave would not reach the center of the shaft, therefore, in the center of the shaft, moment of elasticity should be practically equal to zero. This conclusion was 


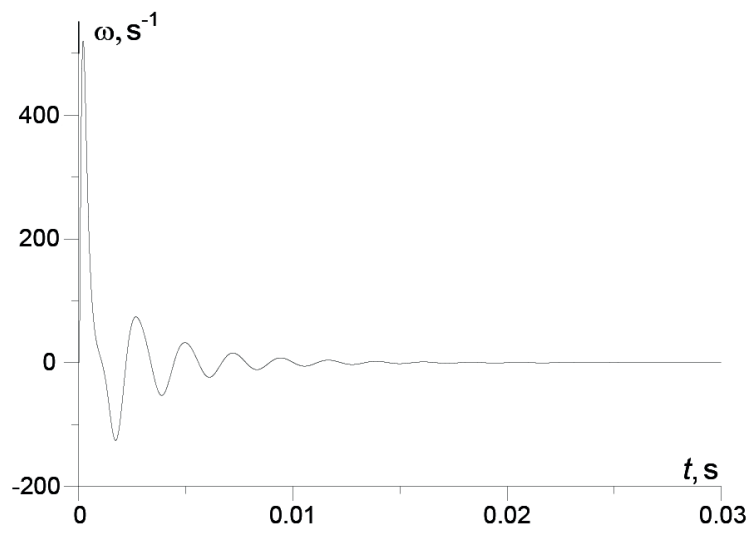

Figure 4 Angular velocity the shaft's torsion in the point of discretization $N o=9$ (0.6m from the left end of the shaft) in terms of time (non-linear variant)

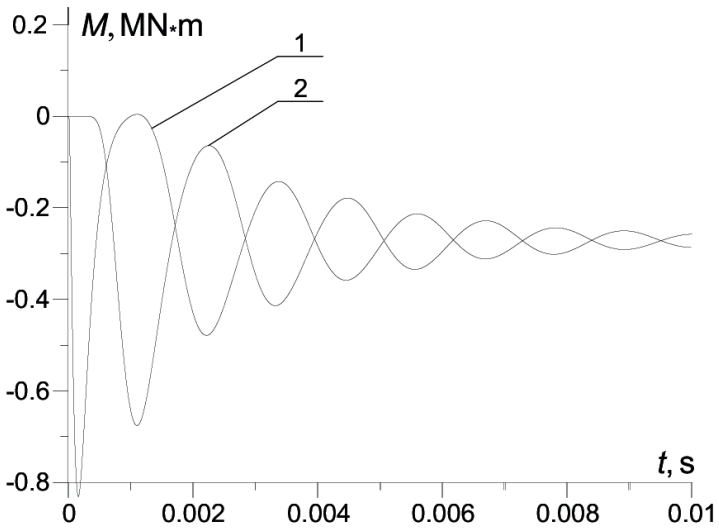

Figure 5 Moment of elasticity in a shaft in terms of time: 1 - left end of the shaft (No 3-4), 2 - center of the shaft (No 45-46)

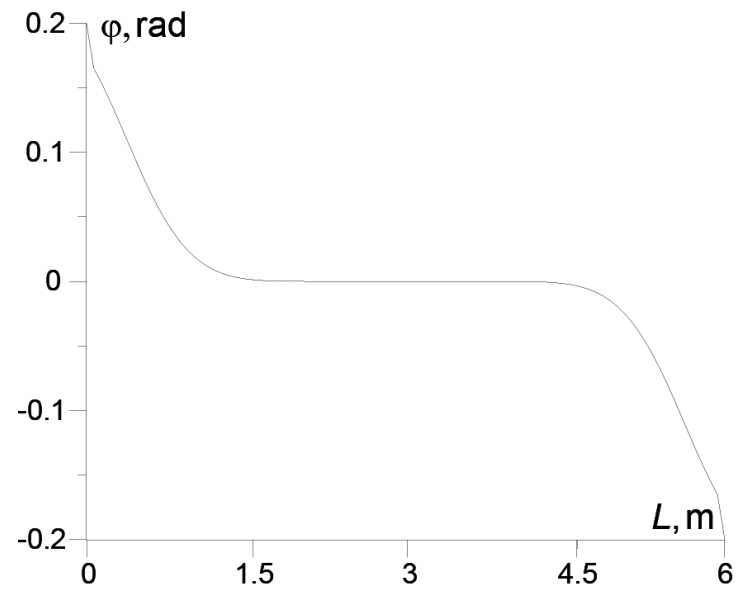

Figure 6 Angle of torsion of the shaft in $v$ of its length

$$
t=0.2 \mathrm{~ms}
$$

confirmed in Figure 5. It must also be added that torsion of the ends of the shaft resulted in high values of amplitude of rotational speed, and there are no such values in the central part. The situation in the next pair of analysed figures (Figure 7 and 9) is different. Time increased 10 times here. The wave reached the center of the shaft and function of the torsions angle of the shaft's discrete parts in an argument of spatial coordinate approaches to linear value; whereas

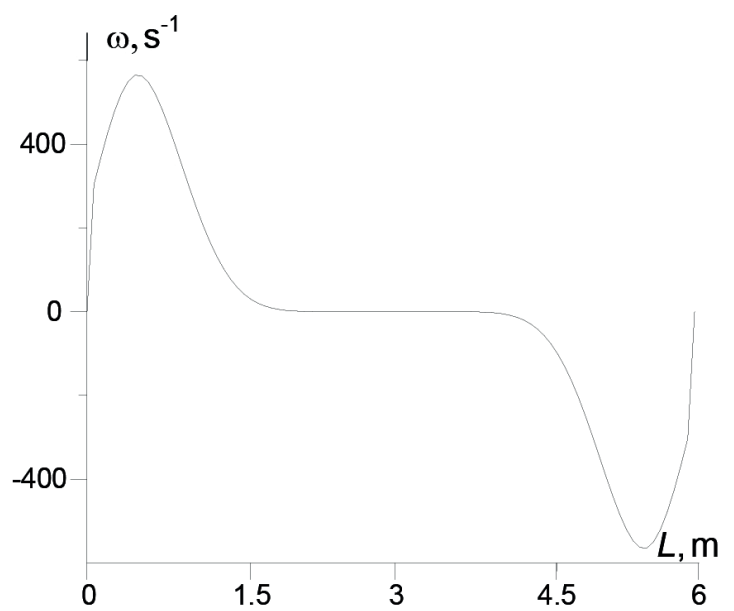

Figure 7 Angular velocity of the shaft in terms of its length $t=0.2 \mathrm{~ms}$

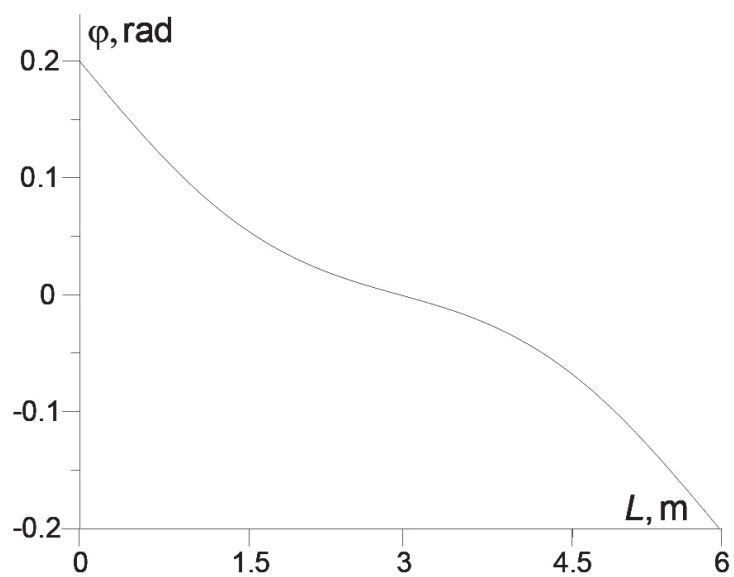

Figure 8 Angle of torsion of the shaft in terms of its length $t=2 \mathrm{~ms}$

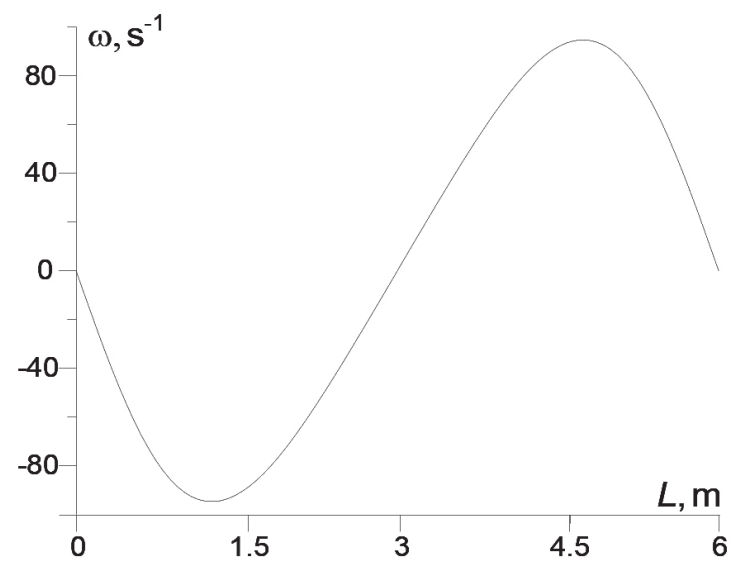

Figure 9 Angular velocity of the shaft in terms of its length

$$
t=2 \mathrm{~ms}
$$

function of velocity of torsion of the shaft approaches to zero (it should be emphasized that time derivative from an angle of torsion is rotational speed). Therefore, it is obvious that in steady state, (about $\mathrm{t}=0.02 \mathrm{~s}$ ) the shaft will be linearly twisted, (see Figures 2 and 3).

Figures 10 to 12 show spatiotemporal behaviour of the functions in a three-dimensional system. Figure 10 shows spatiotemporal distribution of function of an angle 


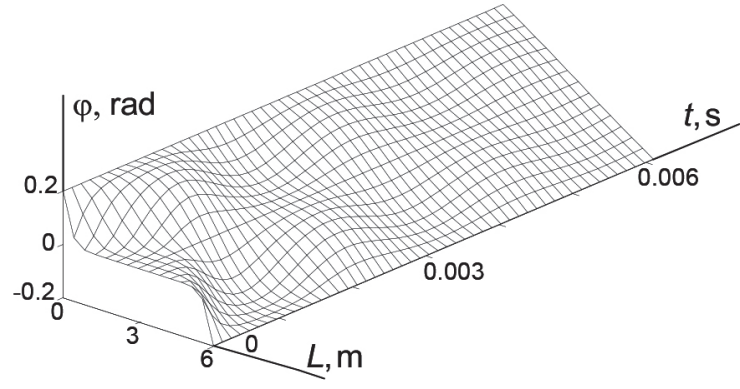

Figure 10 Spatiotemporal distribution of function of the shaft's torsion angle in a time range $t \in[0 ; 0.006] s$

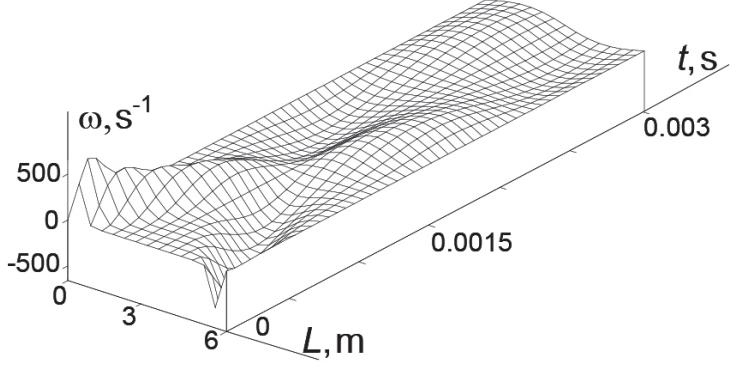

Figure 11 Spatiotemporal distribution of function of shaft's angular velocity in a time range $t \in[0 ; 0.003] s$

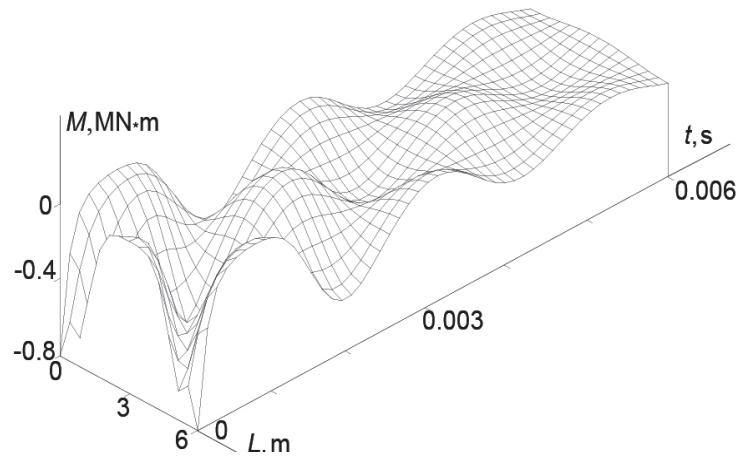

Figure 12 Spatiotemporal distribution of function of the shaft's moment of elasticity in a time range $t \in[0 ; 0.006] \mathrm{s}$

of torsion of the shaft in a time range $t \in[0 ; 0.006] \mathrm{s}$; and Figure 11 shows spatiotemporal distribution of function of angular velocity of the shaft in a time range $t \in[0 ; 0.003]$ $\mathrm{s}$, and Figure 12 shows spatiotemporal distribution of function of moment of elasticity in the shaft in a time range $t \in[0 ; 0.006] \mathrm{s}$. Figure 10 should be analysed together with the Figures 2, 3 and 6, 8. Figure 11 should be analysed together with Figures 4 and 7, 9. Figure 12 should be analysed together with Figure 5 . What is important is clear visualization of spatiotemporal movement of mechanical wave in an isotropic non-linear environment of continuum of the shaft.

An analysis of subsequent figures (Figures 13 to 20) refers to the second experiment. Figure 13 shows an angle of torsion of the shaft's discretization units in the point No $=9(0.6 \mathrm{~m}$ from the left end $)$ in terms of time (linear variant) and Figure 14 shows an angle of torsion of the shaft in the point $\mathrm{No}=9$ ( $0.6 \mathrm{~m}$ from the left end) in a function of time (1st variant - linear and 2nd variant- non-linear) in a time range $t \in[2.4 ; 2.5] \mathrm{s}$.

With reference to the first experiment, the shaft was twisted and its ends were immobilized (boundary conditions - Heaviside function), in the second experiment, the shaft was quickly twisted and relieved, which resulted in the lack of torsion of the shaft; in steady state. That is, no force affects the shaft within more than $2.5 \mathrm{~s}$, which can be seen in Figure 13. Amplitude of shaft's oscillation slowly vanishes to zero, in contrast with the first experiment (Figure 3). Figure 14 shows a comparative analysis for both cases: 1 - linear and 2 - non-linear. During the analysis of Figures 2 and 3, the causes of propagation of both lines were explained. In the analysed Figure 14, a very important moment of the phase shifts of both graphs should be emphasized. This fact is very important. Analysing the transmissions of movement in a linear variant, data concerning amplitude and phase shift are obtained. Amplitude is not a priority information here, whereas, a phase shift is a significant information, particularly with reference to the precision systems (transmissions of movement with the engines of PMSM and BLDC type [5], in the special-purpose transport systems), in which accurate information about location of a rotor and output shaft of a load mechanism is required.

Figure 15 shows the instantaneous moment of elasticity in two parts of the shaft: between the points of discretization No 2 and No 3 (left end of the shaft), as well as No 45 and No 46 (center of the shaft) in a time range $t \in[0 ; 0.05] \mathrm{s}$. Figure 15 shows that within up to 10 $\mathrm{ms}$, mechanical wave forms very complicated movements that were analysed above (see Figure 5); whereas within more than $10 \mathrm{~ms}$, the situation radically changes, different from the first experiment. Oscillatory fluctuations of both functions are practically identical with a zero phase of oscillation. It means that after $10 \mathrm{~ms}$, function of moment of elasticity along the shaft is practically identical. Figure 16 shows a current moment of elasticity in the shaft between the points of discretization No 45 - 46. The moments of elasticity in all parts of a long shaft have practically the same value.

Figures 17 and 18 show the shaft's torsion angle as a function of length for $\mathrm{t}=2 \mathrm{~ms}$ and angular velocity of the shaft as a function of length for $t=0.2 \mathrm{~ms}$. Figure 17 should be analysed together with Figure 6 and Figure 18 with Figure 10. One may conclude from the comparative 


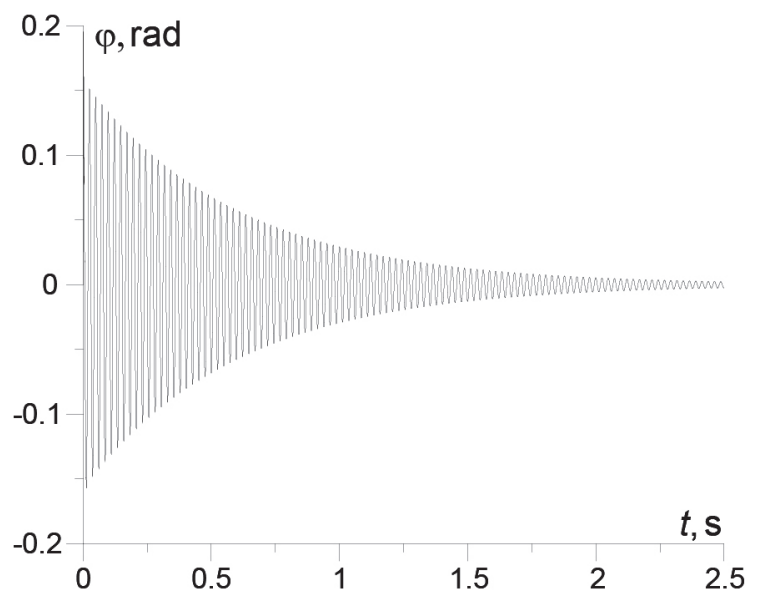

Figure 13 Angle of torsion of the shaft in the point $N o=9(0.6 \mathrm{~m}$ from the left end) in a function of time (linear variant)

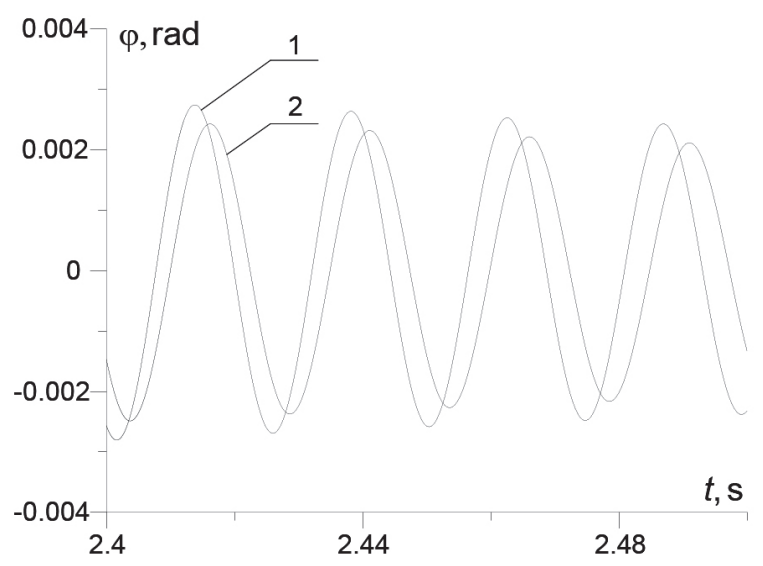

Figure 14 Angle of torsion of the shaft in the point No $=9$ ( $0.6 \mathrm{~m}$ from the left end) in a function of time (1 variant linear, 2 - non-linear variant)

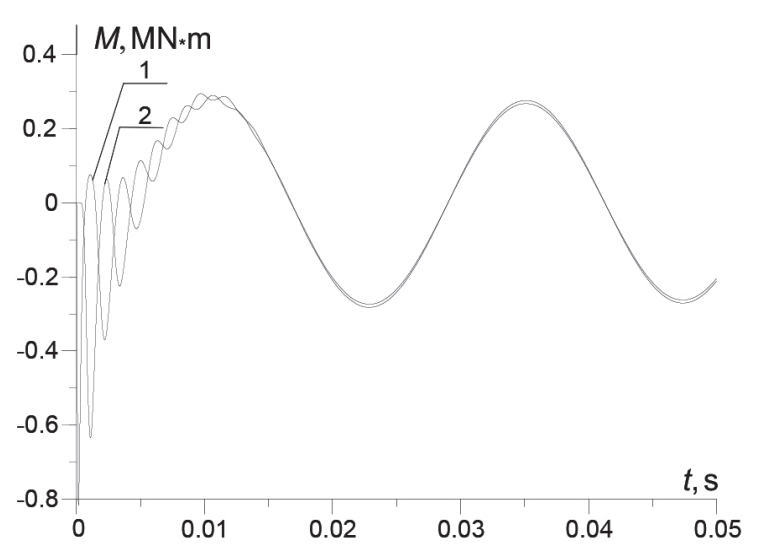

Figure 15 Moment of elasticity in the shaft as a function of time 1 - left end of the shaft (No 2-3), 2 - center of the shaft (No 45-46)

analysis that the wave processes for both experiments are visually similar. However, one important moment must be emphasized. In the first experiment, function of the torsions angle approaches to linear relation significantly faster in comparison to the second experiment. This means that oscillation of the shaft's ends (second experiment) triggers

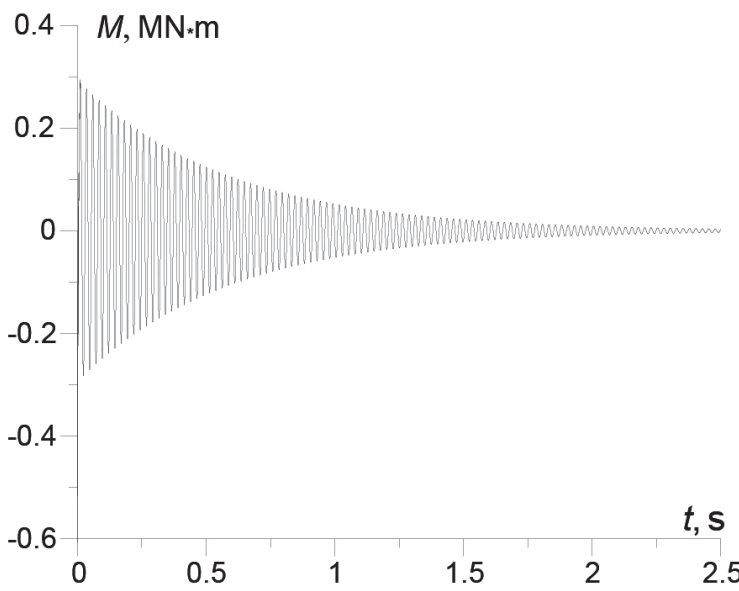

Figure 16 Moment of elasticity in the center of the shaft as a function of time (No 45 - 46)

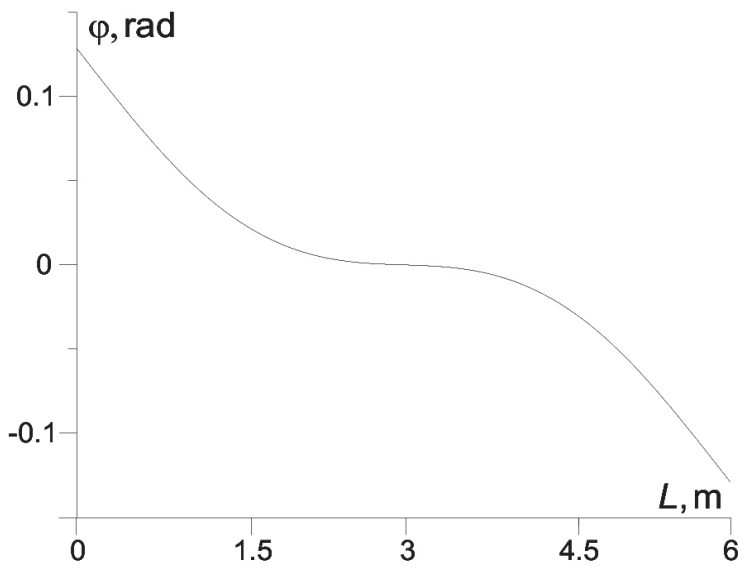

Figure 17 Angle of torsion of the shaft as a function of length $t=2 \mathrm{~ms}$

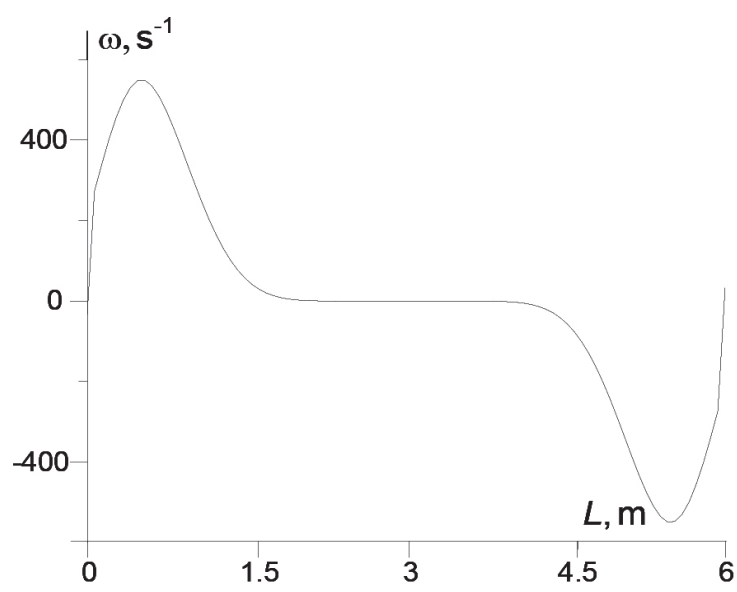

Figure 18 Angular velocity of the shaft as a function of length $t=0.2 \mathrm{~ms}$

an effect of the elastic wave braking. It shows that it is necessary to analyse the long transmissions of movement at the level of analytical mechanics of the continuous systems, that is, at the level of the field systems of distributed parameters $[1,2,17]$.

Figures 19 and 20 show spatiotemporal distributions 


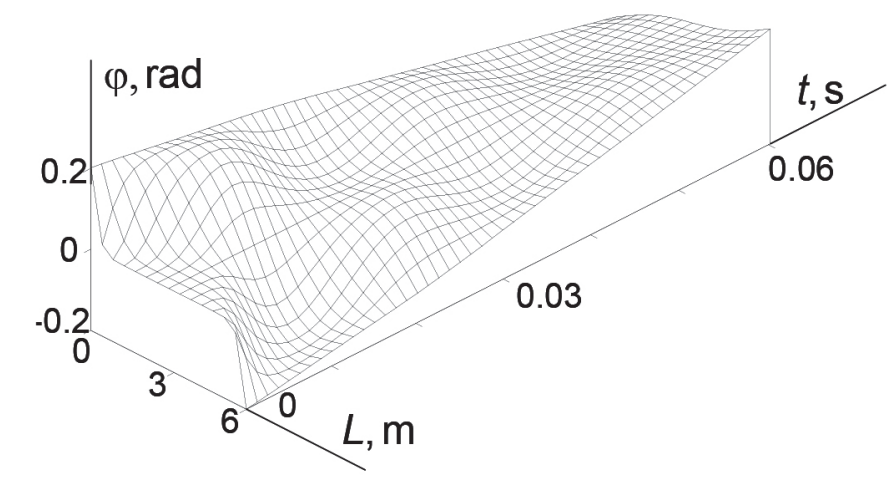

Figure 19 Spatiotemporal distribution of function of the shaft's torsion angle in a time range $t \in[0 ; 0.006] \mathrm{s}$

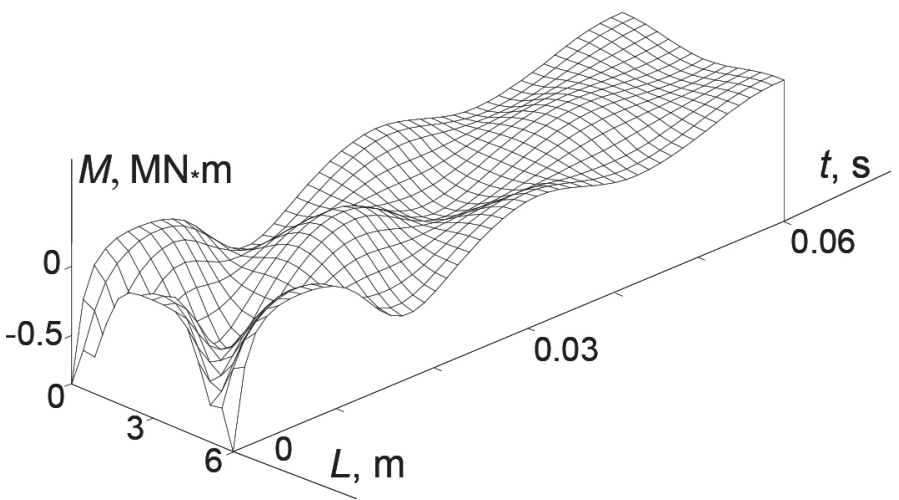

Figure 20 Spatiotemporal distribution of function the

of function of the torsion angle and function of velocity of this angle in a time range $t \in[0 ; 0.006] \mathrm{s}$. An analysis of Figures 19 and 20, including Figures 10 and 12, confirms again an effect of braking of mechanical wave in the second experiment with reference to the first one. Comparative analysis shows fivefold change of value of an effect of braking, whereas, the amplitudes of oscillation of analysed functional relations do not differ significantly.

Mentioned fact gives a basis for conclusion of usefulness of using the Dirichlet problem, including boundary conditions using the Heaviside and Dirac function (here of finite value of impulse) in a non-linear relation between tensors of deformation and elongation.

\section{Conclusions}

Use of the modified Hamilton-Ostrogradsky principle, using extended Lagrangian function to develop mathematical models of long transmission shafts, including non-linear relation between tensors of deformation and elongation, extends the possibilities of analysing oscillatory unspecified processes in a transmission of movement of electric drives in the transport tasks.

Conducted experiments showed that in the tasks connected with an analysis of a complex transmission of movement that includes long drive shafts as the systems of distributed parameters, practically all the mechanical states can be analysed: both transitional and fixed. It gives higher accuracy of mathematical calculations of dynamic states of an object in comparison to considering such object in a multi-body system of lumped parameters, analysed as a system of lumped parameters.

While solving wave equation of oscillation torsions of a long shaft, as an element of complex transmission of movement of electric drives, boundary conditions in the form of the Heaviside function (constant value of step function) and Dirac function (finite value of impulse function) can be applied. On the one hand, such an approach simplifies using a numerical method of the spatial discretization of the shaft's state equations (simple method), on the other hand, it allows to obtain sufficient information about physical processes in the complex transmission of movement.

Based on the computer simulation results, it may also be concluded that:

- comparative analysis of both experiments, connected with examining a long shaft that includes linear and non-linear relation between tensors of deformation and elongation, shows significant difference for both experiments - concerning various frequencies and amplitude of oscillation of the torsion angle, as well as velocity of the shaft's torsion;

- in the first millisecond of a transitional process of the shaft's oscillations, it can be seen that mechanical wave does not move very quickly along continuum of the shaft. The movement starts from the ends of the shaft and moves towards its center. It causes an effect of counterphase that decreases with elapse of time;

- angular motion of the shaft's transmission causes an effect of braking of moving mechanical wave along 
continuum of the shaft in comparison to the case when the ends of the shaft are inhibited;

- $\quad$ phase shift of the shaft's rotation angle that emerges in both experiments connected with examination of shaft's movement transmission in a linear and nonlinear system is a very important information. Slight deviations the shaft's rotation angle from the real value results in giving a control signal at the wrong moment in the automated control drives [4, 14];

- results of simulation in the 3D format are of informative value and can be applied to spatiotemporal distributions of analysed functions.

\section{References}

[1] WANG, J., LIU, Y., SUN, C. Adaptive neural boundary control design for nonlinear flexible distributed parameter systems. IEEE Transactions on Control Systems Technology [online]. 2018, p. 1-15. ISSN 1063-6536, eISSN 1558-0865. Available from: https://doi.org/10.1109/TCST.2018.2849072

[2] WANG, Z., WU, H., HAN, K. Sampled-data control for linear time-delay distributed parameter systems. ISA Transactions [online]. 2018, 92, p. 75-83. ISSN 0019-0578. Available from: https://doi.org/10.1016/j.isatra.2019.02.002

[3] POPENDA, A. Mathematical modelling of transmission shafts based on electrical and mechanical similarities. Przeglad Elektrotechniczny. 2019, 12, p. 196-199. ISSN 0033-2097, eISSN 2449-9544.

[4] ORLOWSKA-KOWALSKA, T., SZABAT, K. Control of the driver system with stiff and elastic couplings using adaptive neuro-fuzzy approach. IEEE Transactions on Industrial Electronics [online]. 2007, 54(1), p. 228-240. ISSN 0278-0046. Available from: https://doi.org/10.1109/TIE.2006.888787

[5] CHABAN, A. Principle Hamilton-Ostrogradski in electromechanical systems. Lviv, 2015. ISBN 978-966-2598-46-9.

[6] KUCERA, L., GAJDAC, I., MRUZEK, M. Simulation of parameters influencing the electric vehicle range. Communications - Scientific Letters of the University of Zilina [online]. 2016, 18(1A), p. 59-63. ISSN 1335-4205, eISSN 2585-7878. Available from: http://komunikacie.uniza.sk/index.php/communications/article/view/360

[7] KORENCIAK, D., GUTTEN, M., ADAMEC, J., GLOWACZ, A., CICHY, A. Analysis of engine knocksensor. Communications - Scientific Letters of the University of Zilina [online]. 2018, 20(1), p. 37-41. ISSN 1335-4205, eISSN 2585-7878. Available from: http://komunikacie.uniza.sk/index.php/communications/article/view/43

[8] LEWINSKI, A., TORUN, A., PERZYNSKI, T. Risk analysis as a basic method of safety transmission system certification. In: Modern Transport Telematics TST 2011: proceedings [online]. Vol 239. Communications in Computer and Information Science. Berlin, Heidelberg: Springer, 2011. ISBN 978-3-642-24659-3, eISBN 978-3-642-24660-9, p. 47-53. Available from: https://doi.org/10.1007/978-3-642-24660-9_6

[9] LEWINSKI, A., PERZYNSKI, T. Telematics as a new method of transport system safety verification. In: Research Methods and Solutions to Current Transport Problems ISCT21 2019: proceedings [online]. Vol 1032. Advances in Intelligent Systems and Computing. Cham: Springer, 2020. ISBN 978-3-030-27686-7, eISBN 978-3-030-27687-4. Available from: https://doi.org/10.1007/978-3-030-27687-4_27

[10] BOJIC, N., NIKOLIC, R., BANIC, M., HADZIMA, B. Evaluation of mechanical properties of the two PVC conveyor belts. Communications - Scientific Letters of the University of Zilina [online]. 2018, 20(4), p. 47-51. ISSN 1335-4205, eISSN 2585-7878. Available from: https://doi.org/10.26552/com.C.2018.4.47-51

[11] LUKASIK Z., CZABAN A., SZAFRANIEC, A. Mathematical model of asynchronous pump drive with distributed mechanical parameters. Przeglad Elektrotechniczny. 2018, 6, p.155-159. ISSN 0033-2097, eISSN 2449-9544.

[12] LIS, M., SZAFRANIEC, A. Analysis of transient processes in a drive system with vertical pumps of susceptible motion transmission (in Polish). Przeglad Elektrotechniczny. 2019, 8, p. 21-24. ISSN 0033-2097, eISSN 2449-9544.

[13] CZABAN A., LIS, M. The use of the Hamilton formalism for modelling of power systems with a synchronous motors and susceptible transmission of mechanical power (in Polish). Przeglad Elektrotechniczny. 2018, 1, p. 21-24. ISSN 0033-2097, eISSN 2449-9544.

[14] CZABAN, A., LIS, M. Application of an artificial neural network for determination of rotor current in a driver system: a PMSM motor - a DC generator (in Polish). Przeglad Elektrotechniczny. 2014, 6, p. 272 - 274. ISSN 0033-2097, eISSN 2449-9544.

[15] RABOTNOW, J. Mechanics of deformed bodies (in Russian). Vol. 3. URSS, 2019, ISBN 978-5-9710-5637-9.

[16] WASHIZU, K. Variational methods in elasticity and plasticity. Kittery, ME, U.S.A.: Pergamon Press, 1982. ISBN 9780080267234 .

[17] PUKACH, P. Y. Qualitative methods for the investigation of a mathematical model of nonlinear vibrations of a conveyer belt. Journal of Mathematical Sciences [online]. 2014, 198(1), p. 31-38. ISSN 1072-3374, eISSN 1573-8795. Available from: https://doi.org/10.1007/s10958-014-1770-x

[18] WHITE, D. C., WOODSON, H. H. Electromagnetic energy conversion. New-York: John Wiley \& Sons Inc, 1958. ISBN 978-1124129310. 
[19] ORTEGA, R., LORIA PEREZ, A., NICKLASSON, P. J., SIRA-RAMIREZ, H. Passivity-based control of EulerLagrange systems: mechanical, electrical and electromechanical applications. London: Springer Verlag, 1998. ISBN 978-1-4471-3603-3.

[20] SPORYKHIN, A. N. Hamilton-Ostrogradski principle in the theory of nonlinear elasticity with the combined approach. International Applied Mechanics [online]. 1995, 31, p. 294-297. ISSN 1063-7095, eISSN 1573-8582. Available from: https://doi.org/10.1007/BF00846778

[21] ELSGOLTS, L. Differential equations and the calculus of variations. Moscow: Mir publishers,1977.

[22] YABUKI, A., OHISHI, K., MIYAZAKI, T., YOKOKURA, Y. Force control including contact process using accelerationsensor-based instantaneous state observer for high-stiffness gear drive. In: IEEE 25th International Symposium on Industrial Electronics: proceedings [online]. 2016. eISSN 2163-5145, p. 651-656. Available from: https://doi.org/10.1109/ ISIE.2016.7744966

[23] SZAFRANIEC, A., Mathematical model of asynchronous pump drive and power transformer drive system with complex motion transmission. In: 13th International Scientific Conference Control of Power Systems: proceedings. 2018. p. 104-109.

[24] XU, S., SUN, G., CHENG, Z. Fractional order modeling and residual vibration suppression for flexible two-mass system. In: 29th Chinese Control and Decision Conference CCDC 2017: proceedings [online]. 2017. eISSN 1948-9447, p. 3658-3664. Available from: https://doi.org/10.1109/CCDC. 2017.7979140 